\title{
Capon-Based Single-Snapshot DOA Estimation in Monostatic MIMO Radar
}

\author{
Aboulnasr Hassanien*, Moeness G. Amin, Yimin D. Zhang, and Fauzia Ahmad \\ Center for Advanced Communications, Villanova University, Villanova, PA 19085, USA
}

\begin{abstract}
We consider the problem of single snapshot direction-of-arrival (DOA) estimation of multiple targets in monostatic multiple-input multiple-output (MIMO) radar. When only a single snapshot is used, the sample covariance matrix of the data becomes non-invertible and, therefore, does not permit application of Capon-based DOA estimation techniques. On the other hand, low-resolution techniques, such as the conventional beamformer, suffer from biased estimation and fail to resolve closely spaced sources. In this paper, we propose a new Capon-based method for DOA estimation in MIMO radar using a single radar pulse. Assuming that the angular locations of the sources are known a priori to be located within a certain spatial sector, we employ multiple transmit beams to focus the transmit energy of multiple orthogonal waveforms within the desired sector. The transmit weight vectors are carefully designed such that they have the same transmit power distribution pattern. As compared to the standard MIMO radar, the proposed approach enables transmitting an arbitrary number of orthogonal waveforms. By using matched-filtering at the receiver, the data associated with each beam is extracted yielding a virtual data snapshot. The total number of virtual snapshots is equal to the number of transmit beams. By choosing the number of transmit beams to be larger than the number of receive elements, it becomes possible to form a full-rank sample covariance matrix. The Capon beamformer is then applied to estimate the DOAs of the targets of interest. The proposed method is shown to have improved DOA estimation performance as compared to conventional single-snapshot DOA estimation methods.
\end{abstract}

Keywords: Direction-of-arrival estimation, single snapshot, Capon estimator, MIMO radar

\section{INTRODUCTION}

Direction-of-arrival (DOA) estimation finds applications in many diverse areas, such as radar, sonar, wireless communications, seismology, radio astronomy, and medical diagnostics [1]. The problem of estimating the DOAs of signals impinging on an array of sensors has been thoroughly investigated for several decades [1], [2]. Recently, the problem of DOA estimation using a single snapshot has attracted considerable attention [3], [4]. Situations where there are only a few data snapshots or even a single snapshot available for DOA estimation are not uncommon in practice [3]-[6]. For example, in smart automotive systems, adaptive cruise control and emergency brake initiation require performing online estimation of the relative distances and speeds with respect to other targets as well as the targets' DOAs [3]. Due to the rapid change of the relative ranges, velocities, and DOAs, a single snapshot could be the only available data for DOA estimation. In the presence of a single data snapshot, the sample covariance matrix becomes rank-deficient and non-invertible. As a result, DOA estimation techniques, such as the Capon method, are rendered inapplicable. Recently, multiple-input multiple-output (MIMO) radar has been the focus of intensive research [7]-[12]. The advantages of MIMO radar with colocated antennas include improved angular resolution and parameter identifiability, increased number of detectable targets, and extended array aperture by virtual sensors. Many algorithms have been developed for DOA estimation in MIMO radar based on a large available number of data snapshots [13]-[18] as well as using a single pulse [18], [19].

In this paper, we introduce a Capon-based method for DOA estimation of multiple targets in MIMO radar using data collected from a single radar pulse. Transmit beamforming is employed to achieve transmit processing gain. A principal transmit weight vector is properly designed to focus the transmit power within a certain desired spatial sector while minimizing the power radiated in the out-of-sector area. Then, a population of weight vectors of same dimensionality and of same transmit power distribution as that of the principal weight vector is generated

\footnotetext{
*aboulnasr.hassanien@villanova.edu
} 
using the transmit radiation pattern invariance method [20], [21]. A subset of the aforementioned weight vectors is selected and used to simultaneously transmit a set of orthogonal waveforms. Matched-filtering the data collected from one radar pulse to the orthogonal transmit waveforms yields a number of virtual data snapshots that equals to the number of orthogonal waveforms. By properly choosing the number of orthogonal waveforms, a full-rank sample covariance matrix can be constructed using the virtual snapshots. This enables the application of Capon-based DOA estimation techniques. We use simulation examples to validate the effectiveness of the proposed Capon-based single-snapshot DOA estimation method.

This paper is organized as follows. In Section 2, we present the signal model and formulate the DOA estimation problem. In Section 3, we present the proposed Capon-based single-snapshot DOA estimation method and briefly review several existing single snapshot DOA estimation methods. Simulation results are provided in Section 4, while conclusions are drawn in Section 5.

\section{PROBLEM FORMULATION}

We assume a MIMO radar system equipped with $M$ transmit colocated antennas arranged in a uniform linear array (ULA) and $N$ receive antennas arranged in an arbitrary linear array. Let $\mathbf{w}$ be the $M \times 1$ principal transmit beamforming weight vector. The main function of $\mathbf{w}$ is to concentrate the transmit power within a certain desired spatial sector $\boldsymbol{\Theta}=\left[\theta_{\min }, \theta_{\max }\right]$ while minimizing the power radiated in the out-of-sector region $\overline{\boldsymbol{\Theta}}$. There are several methods in the literature which can be used for transmit beamforming design (see for example [22], [23] and references therein). Once the principal transmit beamforming weight vector is designed, it can be used to generate a population of weight vectors of same dimensionality and of same transmit power radiation pattern as that of $\mathbf{w}$. Two weight vectors $\mathbf{w}$ and $\mathbf{w}_{q}$ of same dimensionality are said to have the same transmit power radiation pattern if the following radiation pattern invariance property is satisfied

$$
\left|\mathbf{w}^{H} \mathbf{a}(\theta)\right|^{2}=\left|\mathbf{w}_{q}^{H} \mathbf{a}(\theta)\right|^{2}, \quad \theta \in\left[-\frac{\pi}{2}, \frac{\pi}{2}\right],
$$

where $\mathbf{a}(\theta)$ is the $M \times 1$ transmit array steering vector associated with the spatial direction $\theta$ and $(\cdot)^{H}$ stands for the Hermitian. Following the steps given in [20] and [21], a population of $2^{M-1}-1$ weight vectors which satisfy the radiation pattern invariance property (1) can be generated from the principal weight vector $\mathbf{w}$. Let $r_{i}, i=1, \ldots, M-1$ be the roots of the polynomial $f(x)=w_{1}+w_{2} x+\ldots+w_{M} x^{M-1}$ where $w_{m}$ is the $m$ th entry of the principal weight vector $\mathbf{w}$. The transmit radiation pattern can be expressed in terms of $f(x)$ as follows

$$
\left|\mathbf{w}^{H} \mathbf{a}(\theta)\right|^{2}=|f(x)|_{x=e^{-j 2 \pi d \sin \theta}}^{2} .
$$

Note that reflecting each root against the unit circle does not change (2), i.e., the magnitude of the beampattern remains unchanged. Therefore, in addition to $f(x)$, at most $2^{M-1}-1$ different polynomials of the same order can be constructed, depending on the choice of the $M-1$ combination of the roots $r_{i}$ (or $\left.1 / r_{i}^{*}\right), i=1, \cdots, M-1$ employed for polynomial construction. The coefficients associated with each of the $2^{M-1}$ polynomials represent a transmit weight vector that has the same transmit radiation pattern as that of the principal weight vector. Therefore, the transmit weight vector population denoted as $\mathbf{W}_{\mathrm{pop}}=\left\{\mathbf{w}_{1}, \ldots, \mathbf{w}_{2^{M-1}}\right\}$ with the same radiation can be constructed.

Let $\boldsymbol{\Psi}(t)=\left[\psi_{1}(t), \ldots, \psi_{Q}(t)\right]^{T}$ be the $Q \times 1\left(Q \leq 2^{M}\right)$ vector of transmit waveforms, where $\left\{\psi_{q}(t)\right\}, q=1, \ldots, Q$, is a predesigned set of orthonormal waveforms, $t$ is the fast time, i.e., time index within the radar pulse, and $(\cdot)^{T}$ denotes matrix transpose. Let $\mathbf{w}_{q}, q=1, \ldots, Q$, be a set of weight vectors randomly selected from the population $\mathbf{W}_{\text {pop. }}$. Each weight vector is used to form a transmit beam for radiating one of the orthogonal waveforms. Therefore, the baseband representation of the $M \times 1$ transmit signal vector $\mathbf{s}(t)$ can be expressed as a linear combination of the individual orthogonal waveforms, i.e.,

$$
\mathbf{s}(t)=\sqrt{\frac{M}{Q}} \mathbf{W} \mathbf{\Psi}(t),
$$

where $\mathbf{W}=\left[\mathbf{w}_{1}^{*}, \ldots, \mathbf{w}_{Q}^{*}\right]$ is the $M \times Q$ transmit beamforming weight matrix and $(\cdot)^{*}$ denotes complex conjugate. We assume that each column of the transmit weight matrix $\mathbf{W}$ is normalized to have a unit norm, i.e., the total 
transmit power is fixed to $P_{t}=M$. Note that $\mathbf{s}(t)$ is the vector of the baseband signals used on the transmit side of the MIMO radar system. From a practical viewpoint, the baseband signals $\mathbf{s}(t)$ should be constant modulus in order to achieve transmit power efficiency. It is noted, however, that from a receiver perspective, the signal model (3) enables pulse compression using the signal vector $\boldsymbol{\Psi}(t)$ which need not be constant modulus. This permits additional degrees of freedom in designing the individual orthogonal waveforms.

Assuming that $L$ targets are present in a certain range bin, the baseband representation of the $N \times 1$ received signal vector at the output of the receive array is modeled as

$$
\begin{aligned}
\mathbf{x}(t) & =\sum_{l=1}^{L} \alpha_{l} \mathbf{b}\left(\theta_{l}\right) \mathbf{a}^{T}\left(\theta_{l}\right) \mathbf{s}(t-\tau)+\mathbf{x}_{i}(t)+\mathbf{n}(t) \\
& =\sqrt{\frac{M}{Q}} \sum_{l=1}^{L} \alpha_{l} \mathbf{b}\left(\theta_{l}\right) \mathbf{a}^{T}\left(\theta_{l}\right) \mathbf{W} \mathbf{\Psi}(t-\tau)+\mathbf{x}_{i}(t)+\mathbf{n}(t), \quad 0 \leq t \leq T_{p}
\end{aligned}
$$

where $\alpha_{l}$ is the reflection coefficient associated with the $l$ th target, $\mathbf{b}(\theta)$ is the $N \times 1$ receive steering vector, $\tau$ is the time delay associated with the range bin of interest, $\mathbf{x}_{i}(t)$ is the interference term which summarizes all signal components due to echoes from targets located outside the range bin of interest, $\mathbf{n}(t)$ is the $N \times 1$ vector of additive white Gaussian noise with zero mean and covariance $\sigma_{z}^{2} \mathbf{I}_{N}, \mathbf{I}_{N}$ denotes the $N \times N$ identity matrix, and $T_{p}$ is the radar pulse duration. In (4), the reflection coefficient $\alpha_{l}, l=1, \ldots, L$ are assumed to obey the Swerling II target model, i.e., they remain constant during the entire width of the radar pulse.

The problem can be formulated as follows. Given the data collected at the output of the receive array due to one radar pulse, estimate the target DOAs $\theta_{l}, l=1, \ldots, L$.

\section{SINGLE-SNAPSHOT DOA ESTIMATION}

In this section, we introduce the proposed Capon-based single-snapshot DOA estimation method. We also briefly review three existing methods, namely, the traditional MIMO radar based Capon, the conventional (Bartlett) beamformer, and the spheroidal sequences based DOA estimation, which can be applied to the single-snapshot case. Each of the three existing methods can be viewed as a special case of our proposed formulations.

\subsection{Proposed Capon-based DOA Estimation}

Matched-filtering the received data (4) to a delayed version of each of the orthogonal waveforms yields the virtual data snapshots

$$
\begin{aligned}
\mathbf{x}_{q} & =\int_{\operatorname{Tp}} \mathbf{x}(t) \psi_{q}^{*}(t-\tau) d t \\
& =\sqrt{\frac{M}{Q}} \sum_{l=1}^{L} \alpha_{l}\left(\mathbf{w}_{q}^{H} \mathbf{a}\left(\theta_{l}\right)\right) \mathbf{b}\left(\theta_{l}\right)+\mathbf{n}_{q}, \quad q=1, \ldots, Q,
\end{aligned}
$$

where $\mathbf{n}_{q}=\int_{\mathrm{Tp}} \mathbf{n}(t) \psi_{q}^{*}(t) d t$ is the $N \times 1$ noise vector at the output of the matched filter which has the same statistics as that of $\mathbf{n}(t)$. It is worth noting that the term $\mathbf{x}_{i}(t)$ is filtered out during the matched-filtering step as a result of the orthogonality condition

$$
\int_{\mathrm{Tp}} \psi_{q}(t-\tau) \psi_{p}^{*}\left(t-\tau^{\prime}\right) d t= \begin{cases}1, & q=p, \tau=\tau^{\prime} \\ 0, & \text { otherwise }\end{cases}
$$

Utilizing the virtual data snapshots (5), the $N \times N$ sample covariance matrix can be computed as

$$
\hat{\mathbf{R}}=\frac{1}{Q} \sum_{q=1}^{Q} \mathbf{x}_{q} \mathbf{x}_{q}^{H} .
$$


Assume that the number of transmit antennas is larger than or equal to the number of receive antennas, i.e., $M \geq N$. Then, by choosing $Q \geq N$, the sample covariance matrix (7) is guaranteed to be full-rank. This means that the sample covariance matrix is invertible and the Capon principle can be applied for DOA estimation. It is worth noting that, for the case of $N>M$, the virtual data at the output of the matched filters can be re-arranged such that a full-rank sample covariance matrix of size $M \times M$ can be constructed.

The Capon spectrum can then be computed as [1]

$$
P_{\text {Capon }}(\theta)=\frac{\mathbf{b}^{H}(\theta) \mathbf{b}(\theta)}{\mathbf{b}^{H}(\theta) \hat{\mathbf{R}}^{-1} \mathbf{b}(\theta)} .
$$

The DOAs are obtained by searching for the $L$ largest peaks in the Capon spectrum (8).

\subsection{Traditional MIMO Radar Based DOA Estimation}

The essence of the traditional MIMO radar is to radiate omnidirectionally $Q=M$ orthogonal waveforms, one from each transmit antenna. This corresponds to choosing the transmit beamforming weight matrix to be the $M \times M$ identity matrix, i.e., $\mathbf{W}=\mathbf{I}_{M}$. In this case, the virtual snapshots in (5) reduce to

$$
\mathbf{x}_{m}=\sum_{l=1}^{L} \alpha_{l} \mathbf{a}_{[m]}\left(\theta_{l}\right) \mathbf{b}\left(\theta_{l}\right)+\mathbf{n}_{m}, \quad m=1, \ldots, M,
$$

where $(\cdot)_{[m]}$ is the $m$ th entry of a vector. A full-rank covariance matrix can be constructed using the virtual snapshots (9), that is,

$$
\hat{\mathbf{R}}=\frac{1}{M} \sum_{m=1}^{M} \mathbf{x}_{m} \mathbf{x}_{m}^{H}
$$

The sample convariance matrix (10) enables application of Capon-based for DOA estimation. However, comparing (5) and (9), it can be observed that the proposed method enjoys transmit processing gain $\left(\mathbf{w}_{q}^{H} \mathbf{a}\left(\theta_{l}\right)\right)$ while the traditional MIMO radar suffers from decreased signal strength due to the fact that no coherent processing gain is employed. As a result, the latter is expected to have poor DOA estimation performance.

\subsection{Classical SIMO Radar Based DOA Estimation}

In the classical single-input multiple-output (SIMO) radar configuration, a single transmit waveform is employed. Transmit coherent processing gain is achieved using a single transmit beamforming weight vector, i.e., $Q=1$ is used. This case corresponds to choosing the transmit weight matrix $\mathbf{W}$ in the proposed formulation to be a single vector, e.g., the principal weight vector. In such a case, the virtual snapshots (5) reduce to a single data snapshot

$$
\mathbf{x}_{\text {SIMO }}=\sqrt{M} \sum_{l=1}^{L} \alpha_{l}\left(\mathbf{w}^{H} \mathbf{a}\left(\theta_{l}\right)\right) \mathbf{b}\left(\theta_{l}\right)+\mathbf{n} .
$$

By comparing (5) and (11), it is observed that both formulations have the same power distribution pattern. However, the SIMO configuration has a higher signal strength at the receiver as a result of assigning the total transmit power to a single waveform. Despite the high signal strength, the single-snapshot data does not enable high-resolution DOA estimation. Therefore, the conventional (Bartlett) beamformer has to be used for DOA estimation. The DOAs can be obtained by searching for the $L$ highest peaks in the conventional beamformer spectrum, i.e.,

$$
P_{\mathrm{CB}}(\theta)=\frac{\left|\mathbf{b}^{H}(\theta) \mathbf{x}_{\mathrm{STMO}}\right|^{2}}{\mathbf{b}^{H}(\theta) \mathbf{b}(\theta)}
$$




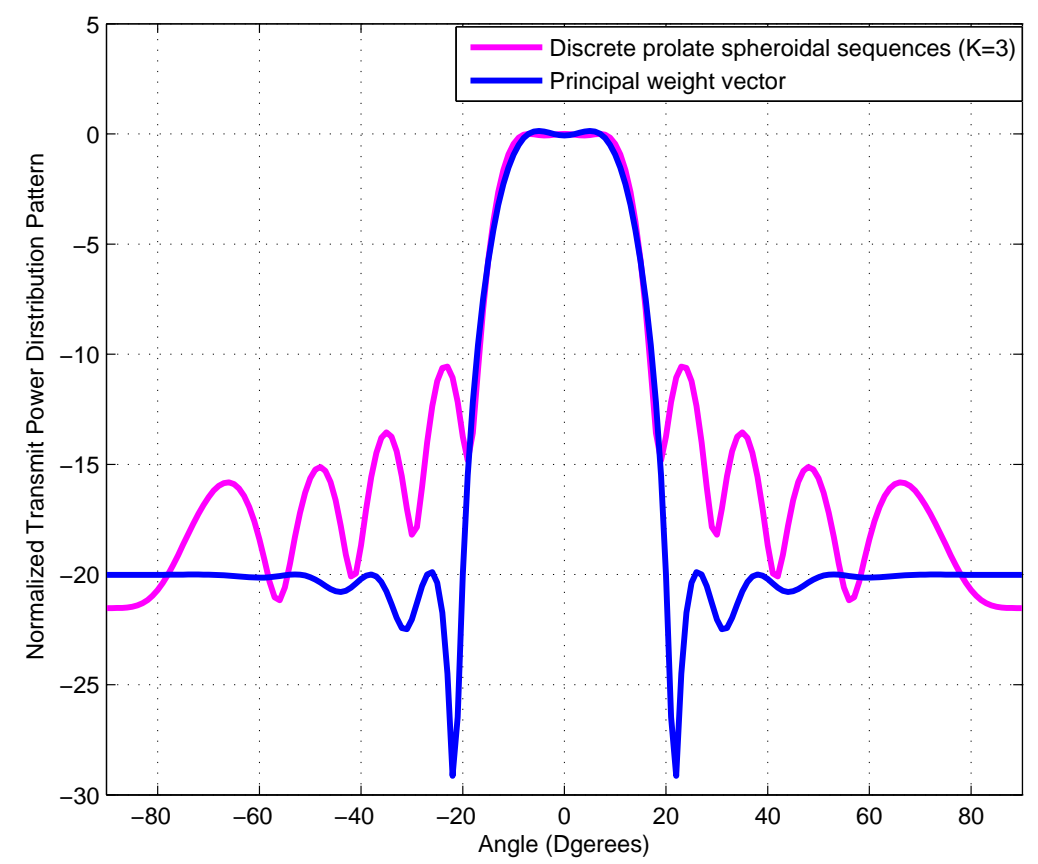

Figure 1. Transmit power distribution versus spatial angle.

\subsection{Spheroidal Sequences Based DOA Estimation}

The discrete prolate spheroidal sequences can be used to incorporate the transmit processing gain at the transmit side of the MIMO radar system. This corresponds to choosing the transmit beamforming weight matrix as $\mathbf{W}=\left[\mathbf{u}_{1}, \ldots, \mathbf{u}_{K}\right]$, where $\mathbf{u}_{k}, k=1, \ldots, K$, are the $K(K<M)$ principal eigenvectors of the positive semidefinite matrix $\mathbf{A}=\int_{\Theta} \mathbf{a}(\theta) \mathbf{a}^{H}(\theta) d \theta$. In this case, the virtual snapshots given in (5) reduce to

$$
\mathbf{y}_{k}=\sqrt{\frac{M}{K}} \sum_{l=1}^{L} \alpha_{l}\left(\mathbf{u}_{k}^{H} \mathbf{a}\left(\theta_{l}\right)\right) \mathbf{b}\left(\theta_{l}\right)+\mathbf{n}_{k}, \quad k=1, \ldots, K .
$$

Since $K$ is small, the $K$ virtual snapshots (13), in general, do not yield a full-rank sample covariance matrix. Similar to the SIMO case, a low-resolution DOA estimation can be performed by searching for the $L$ highest peaks of the spectrum

$$
P_{\mathrm{SPH}}(\theta)=\frac{\mathbf{b}^{H}(\theta) \hat{\mathbf{R}}_{\mathrm{SPH}} \mathbf{b}^{H}(\theta)}{\mathbf{b}^{H}(\theta) \mathbf{b}(\theta)},
$$

where $\hat{\mathbf{R}}_{\mathrm{SPH}}=1 / K \sum_{k=1}^{K} \mathbf{y}_{k} \mathbf{y}_{k}^{H}$.

\section{SIMULATION RESULTS}

The MIMO radar is assumed to have a transmit ULA with $M=12$ antennas spaced half-wavelength apart, and a non-uniform linear receive array of $N=10$ elements. The interelement spacing of the receive antennas are randomly drawn from the range [0 9] measured in units of half-wavelengths. The additive noise signals are modeled as complex Gaussian, zero-mean, and both temporally and spatially white. The targets are assumed to be located within the spatial sector $\boldsymbol{\Theta}=\left[-10^{\circ}, 10^{\circ}\right]$. The principal weight vector is designed by solving the following optimization problem

$$
\begin{aligned}
& \min _{\mathbf{w}} \max _{i}\left|\mathbf{w}^{H} \mathbf{a}\left(\theta_{i}\right)-e^{j \phi\left(\theta_{i}\right)}\right|, \quad \theta_{i} \in \mathbf{\Theta}, i=1, \ldots, I \\
& \text { subject to }\left|\mathbf{w}^{H} \mathbf{a}\left(\theta_{k}\right)\right| \leq \delta, \quad \theta_{k} \in \overline{\mathbf{\Theta}}, \quad k=1, \ldots, K,
\end{aligned}
$$




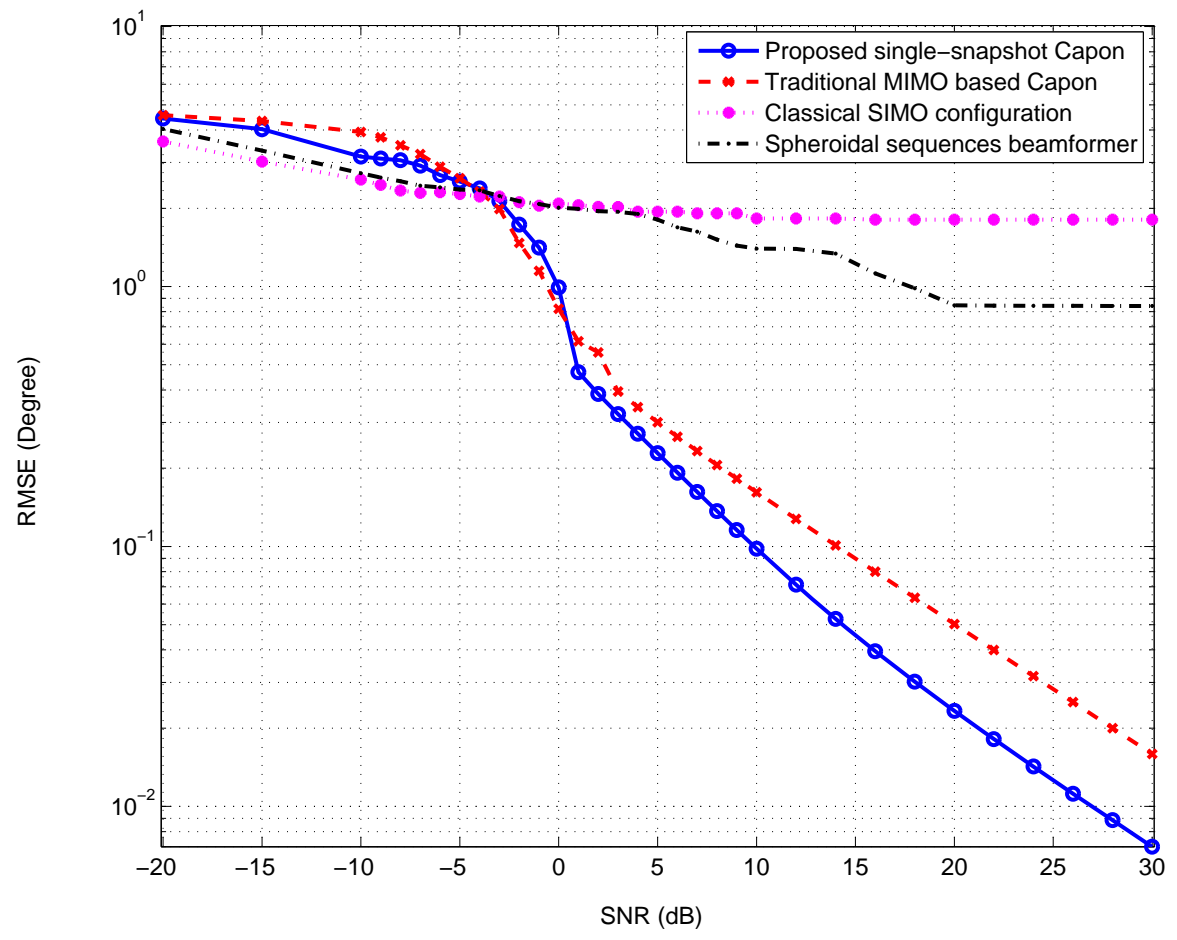

Figure 2. RMSE versus SNR.

where $\theta_{i}$ and $\theta_{k}$ are continuums of angles chosen uniformly or non-uniformly to approximate $\boldsymbol{\Theta}$ and $\overline{\boldsymbol{\Theta}}$, respectively, $\phi(\theta)$ is an arbitrary phase profile which is assumed to be continuous within the desired sector $\boldsymbol{\Theta}$, and $\delta$ is a user-specified positive number to control sidelobe levels. The optimization problem (15) is convex and can be efficiently solved using the interior point methods [24]. The phase profile $\phi(\theta)=2 \pi \sin \theta$ is chosen. The sidelobe levels are controlled by choosing $\delta=\sqrt{0.01}$, i.e., the sidelobe attenuation is at least $20 \mathrm{~dB}$. Following the guidelines of [21], the principal weight vector obtained by solving (15) is used to generate a population of 4095 companion weight vectors. The principal weight vector in addition to the 4095 companion weight vectors form together an overall population of 4096 weight vectors. $Q=12$ weight vectors are drawn randomly from the overall population and used for the proposed single-snapshot DOA estimation. For the conventional beamformer, only the principal weight vector is used at the transmit side. For the spheroidal sequences based method, $K=3$ principal eigenvectors are used. For all methods tested, the total transmit power is fixed to $P_{t}=M$.

Fig. 1 shows the normalized transmit power distribution for the proposed method and the spheroidal sequences based method. For the traditional MIMO configuration, the transmit power distribution is flat due to the onmidirectional transmission of the waveforms and, therefore, is not shown in the figure. For the classical SIMO configuration, the transmit power distribution is the same as that of the proposed method because the employed transmit weight vector is the principal weight vector designed for the proposed scheme.

To test the high resolution capability of the proposed method, two targets are assumed to be located at $-3^{\circ}$ and $3^{\circ}$. Fig. 2 depicts the root-mean square error (RMSE) versus the signal-to-noise ratio (SNR) for all considered methods. The results are averaged over 300 independent runs in each case. We observe that both conventional and spheroidal sequences beamformers have poor performance at moderate and high SNR values, which can be attributed to the low-resolution and biased features of the estimators. The figure also shows that the traditional MIMO based Capon has good RMSE performance especially at high SNR values. Finally, it can be seen from the figure that the proposed Capon-based single-snapshot DOA estimation method has the best RMSE performance as compared to all other methods.

Fig. 3 plots the probability of target resolution versus the SNR for all considered methods. The targets 


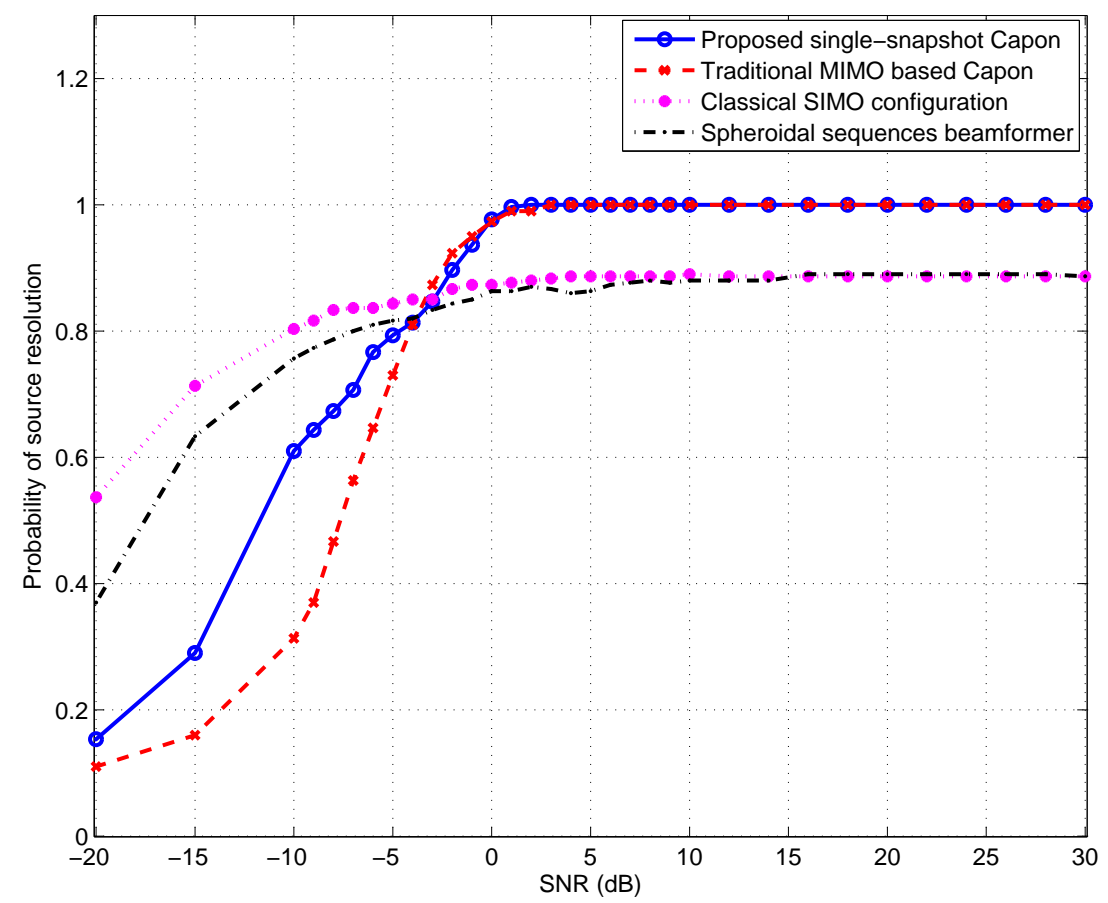

Figure 3. Probability of target resolution versus SNR.

are assumed to be resolved if at least two peaks in the spectrum are observed and the following condition is satisfied $[1]$

$$
\left|\hat{\theta}_{l}-\theta_{l}\right| \leq \frac{\left|\theta_{2}-\theta_{1}\right|}{2} \quad l=1,2
$$

It can be observed from Fig. 3 that the conventional beamformer and the spheroidal sequence based method cannot achieve a good probability of target resolution performance even at very high SNR values mainly due to the low-resolution capability of the two estimators. The proposed Capon-based method and the traditional MIMO based Capon method achieve a 100\% resolution for SNR values higher than $0 \mathrm{~dB}$. For smaller SNR values, the proposed method provides better resolution capabilities as compared to the traditional MIMO based Capon method.

\section{CONCLUSIONS}

The problem of DOA estimation of multiple targets in MIMO radar using a single snapshot has been addressed. A Capon-based single-snapshot DOA estimation method was proposed. Transmit coherent processing gain was achieved by properly designing a principal transmit weight vector to focus the transmit power within the desired spatial sector while minimizing the power radiated in the out-of-sector directions. The designed weight vector was used to generate a population of weight vectors with the same dimensionality and same transmit power distribution as that of the principal vector. The generated population of weight vectors enables transmitting multiple orthogonal waveforms over a single illumination. All waveforms are radiated with the same transmit radiation pattern as that of the principal transmit beamforming weight vectors, i.e., same transmit processing gain is achieved for each waveform. At the radar receiver, the orthogonal waveforms lend themselves to providing a number of virtual data snapshots equal to the number of orthogonal waveforms, thereby permitting the application of Capon-based DOA estimation. The proposed method was shown to outperform the classical MIMO radar based Capon, the conventional (Bartlett) beamformer, and the spheroidal sequences based beamformer. 


\section{REFERENCES}

[1] H. L. V. Trees, Optimum Array Processing. New York: Wiley, 2002.

[2] H. Krim and M. Viberg, "Two decades of array signal processing research: The parametric approach," IEEE Signal Process. Mag., vol. 13, pp. 67-94, Jul. 1996.

[3] P. Häcker and B. Yang, "Single snapshot DOA estimation," Adv. Radio Sci., vol. 8, no. 16, pp. 251-256, 2010.

[4] P. Heidenreich and A. Zoubir, "Computationally simple DOA estimation of two resolved targets with a single snapshot," in Proc. IEEE Int. Conf. Acoust., Speech, Signal Process., Kyoto, Japan, Mar. 2012, pp. 2553-2556.

[5] S. Fortunati, R. Grasso, F. Gini, and M. Greco, "Single snapshot DOA estimation using compressed sensing," in Proc. IEEE Int. Conf. Acoust., Speech, Signal Process., Florence, Italy, May 2014, pp. 2297-2301.

[6] S. Fortunati, R. Grasso, F. Gini, M. Greco, and K. Lepage, "Three CS-based beamformers for single snapshot DOA estimation," in Proc. European Signal Process. Conf., Sept. 2014, pp. 1044-1048.

[7] E. Fishler, A. Haimovich, R. Blum, D. Chizhik, L. Cimini, and R. Valenzuela, "MIMO radar: An idea whose time has come," in Proc. IEEE Radar, Honolulu, HI, Apr. 2004, pp. 71-78.

[8] J. Li and P. Stoica, "MIMO radar with colocated antennas," IEEE Signal Process. Mag., vol. 24, pp. 106114, Sep. 2007.

[9] J. Li and P. Stoica, MIMO Radar Signal Processing. New Jersy: Wiley, 2009.

[10] A. Hassanien and S. Vorobyov, "Phased-MIMO radar: A tradeoff between phased-array and MIMO radars," IEEE Trans. Signal Process., vol. 58, no. 6, pp. 3137-3151, Jun. 2010.

[11] A. Hassanien and S. A. Vorobyov, "Why the phased-MIMO radar outperforms the phased-array and MIMO radars," in Proc. European Signal Process. Conf., Aalborg, Denmark, Aug. 2010, pp. 1234-1238.

[12] A. Hassanien and S. Vorobyov, "Transmit energy focusing for DOA estimation in MIMO radar with colocated antennas," IEEE Trans. Signal Process., vol. 59, no. 6, pp. 2669-2682, June 2011.

[13] I. Bekkerman and J. Tabrikian, "Target detection and localization using MIMO radars and sonars," IEEE Trans. Signal Process., vol. 54, no. 10 pp. 3873-3883, Oct. 2006.

[14] A. Hassanien and S. Vorobyov, "Subspace-based direction finding using transmit energy focusing in MIMO radar with colocated antennas," in Proc. IEEE Int. Conf. Acoust., Speech, Signal Process., Prague, Czech Republic, May 2011, pp. 2788-2791.

[15] A. Khabbazibasmenj, A. Hassanien, and S. Vorobyov, "Transmit beamspace design for direction finding in colocated MIMO radar with arbitrary receive array," in Proc. IEEE Int. Conf. Acoust., Speech, Signal Process., Prague, Czech Republic, May 2011, pp. 2784-2787.

[16] Y. D. Zhang, M. G. Amin, and B. Himed, "Joint DOD/DOA estimation in MIMO radar exploiting timefrequency signal representations," EURASIP Journal on Advances in Signal Process., vol. 2012, pp. 1-10, July 2012.

[17] A. Khabbazibasmenj, A. Hassanien, S. Vorobyov, and M. Morency, "Efficient transmit beamspace design for search-free based DOA estimation in MIMO radar," IEEE Trans. Signal Process., vol. 62, no. 3, pp. 14901500, Mar. 2014.

[18] D. Nion and N. Sidiropoulos, "Tensor algebra and multidimensional harmonic retrieval in signal processing for MIMO radar," IEEE Trans. Signal Process., vol. 58, no. 11, pp. 5693-5705, Nov. 2010.

[19] L. Xu, J. Li, and P. Stoica, "Target detection and parameter estimation for MIMO radar systems," IEEE Trans. Aerosp. Electron. Syst., vol. 44, no. 3, pp. 927-939, July 2008.

[20] A. Hassanien, S. A. Vorobyov, and A. Khabbazibasmenj, "Transmit radiation pattern invariance in MIMO radar with application to DOA estimation," IEEE Signal Process. Lett., vol. 22, pp. 1609-1613, Oct 2015.

[21] A. Khabbazibasmenj, A. Hassanien, and S. A. Vorobyov, "How many beamforming vectors generate the same beampattern?," arXiv preprint, arXiv:1402.1682, 2014.

[22] A. Hassanien and S. Vorobyov, "Transmit/receive beamforming for MIMO radar with colocated antennas," in Proc. IEEE Int. Conf. Acoust., Speech, Signal Process., Taipei, Taiwan, Apr. 2009, pp. 2089-2092.

[23] G. Hua and S. Abeysekera, "MIMO radar transmit beampattern design with ripple and transition band control," IEEE Trans. Signal Process., vol. 61, no. 6, pp. 2963-2974, June 2013.

[24] S. Boyd and L. Vandenberghe, Convex Optimization. Cambridge University Press, 2009. 